\title{
Experiment and analysis of potato-soil separation based on impact recording technology
}

\author{
Zhongcai Wei ${ }^{1}$, Hongwen $\mathrm{Li}^{1 *}$, Yijin Mao ${ }^{2}$, Chuanzhu Sun ${ }^{3}$, Xueqiang $\mathrm{Li}^{4}$, \\ Wenzheng Liu ${ }^{1}$, Guoliang Su${ }^{1}$ \\ (1. College of Engineering, China Agricultural University, Beijing 100083, China; \\ 2. Shenzhen Escope Technology Co., Ltd, Shenzhen 518052, China; \\ 3. School of Mechanical Engineering, Shandong University of Technology, Zibo 255091, China; \\ 4. Shandong Xicheng Agricultural Machinery Science and Technology Co., Ltd., Dezhou 253600, China)
}

\begin{abstract}
In order to discover the damage mechanism and improve separation performance in the separation process of potato-soil mixture, the experiment was conducted on an in-house test-bed. The impact recording device and high-speed camera technology were employed in order to obtain the instantaneous dynamics of the potato-soil mixture for detail data analysis. Five vibration intensities were defined according to the vibration frequency and amplitude. It was found that the mean number of impacts and maximum impact acceleration increased significantly as the level of vibration intensity rose. As a result, the separation performance increased significantly, however, the bruising rate also increased to a certain extent. The mathematical relationship between the maximum impact acceleration and the factors of interest, including vibration amplitude, the vibration frequency and the operating speed of the separation sieves was established through the response surface experiment. It was demonstrated that the presented model was capable to reflect the degree of the factors on influencing bruising rate and separation performance. According to the significance on the maximum impact acceleration, the factors of interest were given in a descending order with vibration frequency, vibration amplitude, running speed of the separation sieve. A set of the optimum operating parameters were found to achieve a desired separation performance as follows, the vibration amplitude was $34.1 \mathrm{~mm}$, the vibration frequency was $5.24 \mathrm{~Hz}$, the running speed of the separation sieve was $2.05 \mathrm{~m} / \mathrm{s}$; where the maximum impact acceleration was $98.62 \mathrm{~g}$, the relative error was $3.23 \%$, the bruising rate was $1.81 \%$ and the separation performance was $98.5 \%$. The presented model can potentially provide a technical reference for further investigation of the separation mechanism and development of measures for reducing the loss of separation.
\end{abstract}

Keywords: potato, impact recording, high speed camera, potato-soil separation, mechanization, movement characteristics DOI: $10.25165 /$ j.ijabe. 20191205.4573

Citation: Wei Z C, Li H W, Mao Y J, Sun C Z, Li X Q, Liu W Z, et al. Experiment and analysis of potato-soil separation based on impact recording technology. Int J Agric \& Biol Eng, 2019; 12(5): 71-80.

\section{Introduction}

Although China has the largest planting area and highest yield of potato in the world, the mechanization level of potato production cannot meet the actual demand $d^{[1-3]}$. Harvesting is the most crucial step of the whole potato production mechanization process, and the most challenging issue during this process is to separate potatoes, soil and impurity thoroughly and meanwhile to control damage rate and bruising rate ${ }^{[4-6]}$. Usually potatoes need to be harvested in a short time. While two-stage harvesting demands manual picking,

Received date: 2018-08-11 Accepted date: 2019-07-23

Biographies: Zhongcai Wei, $\mathrm{PhD}$ candidate, research interests: agricultural mechanization, potato mechanization production technology, Email: weizc2011sdut@163.com; Yijin Mao, Engineer, research interests: agricultural mechanization, Email: ymao.mu@gmail.com; Chuanzhu Sun, Professor, research interests: agricultural mechanization, potato mechanization production technology, Email: suncz@sdut.edu.cn; Xueqiang Li, Engineer, research interests: potato mechanization production technology, Email: 1xqllsd@163.com; Wenzheng Liu, PhD candidate, research interests: agricultural mechanization, potato mechanization production technology, Email: caulwzheng@126.com; Guoliang Su, Engineer, research interests: agricultural mechanization, potato mechanization production technology, Email: suguoliang0101@163.com.

*Corresponding author: Hongwen Li, PhD, Professor, research interests: agricultural mechanization, Email: lhwen@cau.edu.cn. which takes intense labor, yet low harvesting efficiency ${ }^{[7]}$. In order to improve the separation performance of potato-soil and obtain better clod crushing performance, most of the research have focused on vibration and arrangement of the separation device ${ }^{[8,9]}$.

Regarding the bruising and damage control of potato harvest, the harvesting efficiency and quality can be often improved through developing new types of potato-soil separation device, regulating operating parameters of the harvester, controlling the proportion of the potato-soil in the potato-soil mixture and enhancing the seedling killing operation ${ }^{[10-13]}$. Aiming to meet the demand of heavy soil harvesting conditions in the northern China, Lv et al. ${ }^{[14]}$ designed a separation device with two-grade lift chain where the length of the two-grade lift chain is $3.1 \mathrm{~m}$, the forward speed of the machine is $1.2 \mathrm{~m} / \mathrm{s}$ and the speed of the lift chain line is $1.5 \mathrm{~m} / \mathrm{s}$, and exposed potato rate is $98.1 \%$ and the damage rate is $1.1 \%$. In order to improve the effectiveness of separation of potato combined harvester, Yang et al. ${ }^{[15]}$ designed a vertical circular separation transport device that has the average damage rate of $1.46 \%$ and impurity rate of $2.57 \%$. Feng et al. ${ }^{[16]}$ investigated the impact of the potatoes damage on the coefficient of restitution that is caused by the fall height, the impact contact material and the water content of the potatoes and identify the main factors affecting the damage of potatoes. Owing to the large amount mixture of potato-soil and its strong randomness, the 
separation performance and harvest quality can be easily affected by multiple factors that are related to agricultural machinery and agronomy, hence it is difficult to obtain high efficiency separation and low loss harvest simultaneously ${ }^{[17-19]}$.

Impact recording sensor is often used to evaluate potential damage in mechanized harvesting, cleaning, grading and processing of agricultural products. It has been applied to the bruising assessment of blueberry ${ }^{[20]}$, sugar orange ${ }^{[21]}$, apple ${ }^{[22]}$, potato $^{[23]}$ and other agricultural products. Three-dimensional acceleration sensor and pressure sensor are commonly adopted to collect characteristics of impact and pressure of harvested products. All objectives of the studies above are reducing quality loss of agricultural products through decreasing number and intensity of impact in potato harvesting process ${ }^{[23]}$. Although there were a number of scientific studies on acquisition of impact information with impact detection sensor, investigations on exploring the dynamic characteristics of potato-soil mixture with varying vibration parameters is rare.

Potato collision usually takes place many times on separation sieves during the harvesting process, where high rate of rupture, damage and bruising can occur, leading to a great economic loss to the potato growers. Therefore, a fundamental research on the mechanism of the potato damage is emerging ${ }^{[24]}$. However, it is extremely difficult to carry out a systematical experiment to acquire ideal results due to complexity of the system with a number of variables based on existing potato harvester. For this reason, the work on optimizing and improving the structure of potato harvester and reducing the loss during mechanized harvesting process are strongly limited. A new separation device of potatoseparation for a potato harvester with two vibration intensity adjusting device is developed. The objectives of this preliminary study are as follows: (1) Potato-soil separation experiment under different vibration intensity conditions was conducted to improve the harvesting quality. (2) Response surface analysis experiments were conducted to achieve a desired separation performance. (3) The technology of impact recording and high-speed camera were utilized to provide technical record for further investigation of the separation mechanism and the mathematical relationship between the maximum impact acceleration and the factors of interest, including vibration amplitude, the vibration frequency and the operating speed of the separation sieves was established.

\section{Test-bed structure and working principle}

\subsection{Basic structure}

The separation test-bed used in this study has three parts which are manufactured separately, including the potato-soil separation device (main platform), the feeding device (auxiliary platform) and receiving box (Figure 1).

The main testbed primarily consisted of separation sieve, vibration driving device, driving device II, driving device III, guide slot and adjustment device of transfer angle. The auxiliary platform primarily consisted of feeding device and driving device I. The separation device of potato-soil is used to adjust multiple separation parameters for testing. The feeding device is placed at the feeding end of main platform for the usage of conveying the potato-soil mixture to the main platform with a certain thickness and speed according to test requirements. The receiving box is used to collect the mixture after test. Other than that a rubber pad is employed to cover the upper surface of the guide groove in order to minimize the loss. Due to the fact that it is not suitable to accurately evaluate the multiple factor effects on the existing potato harvester, a test-bed that allows for adjustment of the vibration intensity, vibration frequency, running speed of the separation sieve, tilt angle of separating sieve, and the feeding speed of the potato-soil, effective analysis of the separation performance should be set up. Aiming for developing effective means in improving the separation performance and ensuring harvest quality at the same time, the present experimental work is conducted on such a test-bed. For a normal operation of the test-bed, three driving devices are installed. The driving device I supplies power for the conveyor belt. The driving device II imposes exciting force on the separation sieve by the rubber wheel on the jitter shaft. The driving device III provides the power for the running of separation sieve. Flexible cushion was placed at the bottom of the receiving box during the experiments.

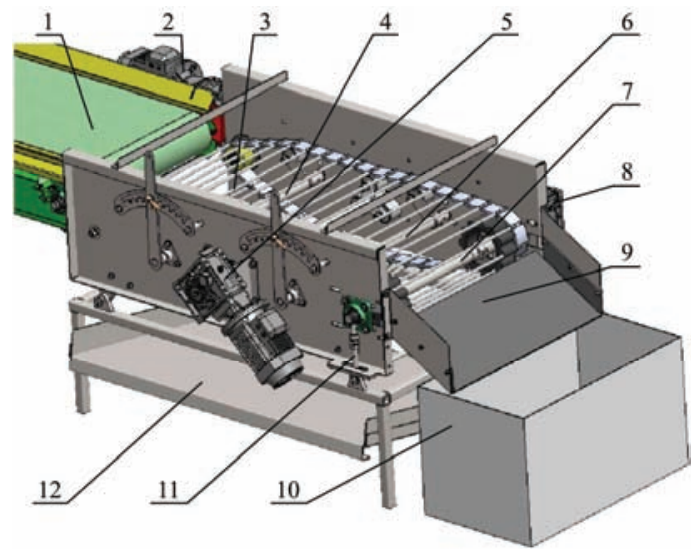

1. Feeding device 2. Driving device I 3. Separation sieve 4. Front amplitude adjustment shaft 5. Driving device II 6. Rear amplitude adjustment shaft 7. Drive shaft of separation sieve 8. Driving device III 9. Guide slot 10. Receiving box 11. Adjustment device of transfer angle 12. Impurity box

Figure 1 Structural diagram of test-bed for the separation of potato-soil

\subsubsection{Separation device of potato-soil}

The key part of the test-bed is the separation device of potato-soil, which encompasses reversing rubber wheel, separation sieve, vibration device, driving device of separation sieve, and the front and rear amplitude adjustment devices (Figure 2). Due to the fact that the front side of the sieves has large amount of mixture while it is decreasing on the end side, two amplitude adjustment handles is put into place to control the degree of draping or supporting. At the same time, the adjustment of the vibration intensity is achieved by regulating the vibration frequency of the jitter rubber wheel of the frequency converter.

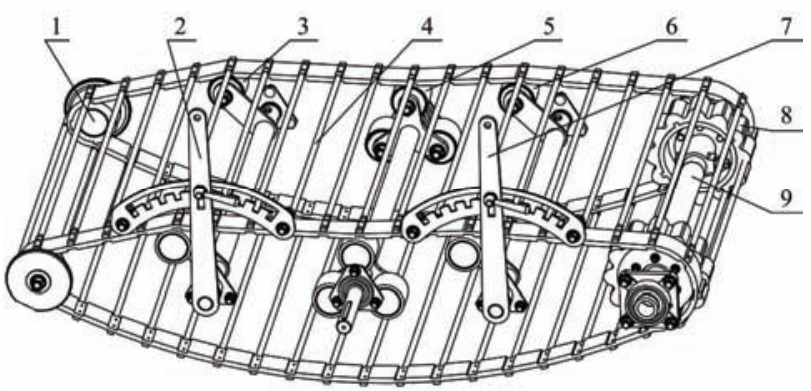

1. Reversing rubber wheel 2. Front amplitude adjustment handle 3. Front adjustment rubber wheel 4. Separation sieve 5. Jitter rubber wheel 6 . Rear adjustment rubber wheel 7. Rear amplitude adjustment handle 8. Plum blossom wheel 9. Drive shaft of separation sieve

Figure 2 Structural diagram of separation device of potato-soil 
According to the cultivation of agronomy and growing depth of potatoes, the thickness for the material layer is $15-20 \mathrm{~mm}$, and the arrangement length of the mixture on the feeding device is $4.5 \mathrm{~m}$. It is desired that the processing amount of the mixture of potato-soil should be about $200-250 \mathrm{~kg} / \mathrm{s} \cdot \mathrm{m}$. The key to the design of test-bed is to take advantage of the characteristics of the bar screen and the vibration imposed by the device, hence ensuring its capacity to process the massive potato-soil mixture.

The running speed of the separation sieve $v_{f}$ is the key parameter of the separation device of potato-soil. On one hand, the higher the running speed of the separation sieve is, the poorer the screening effect of the soil and other impurities could be; on the other hand, separation performance can reduce by lowering running speed of the separation sieve ${ }^{[25]}$. Assuming that the running speed of the potato-soil mixture with the feeding device is $v_{s}$, and the ratio of the running speed of the feeding device and the separation sieve can be expressed by $\lambda=v_{s} / v_{f}$. According to the design manual of agricultural machinery, the general value of $\lambda$ is $0.8-2.5$. In the state of a dry and hard soil with low water content, the separation performance decreases when the running speed of the separation sieves is more than $2 \mathrm{~m} / \mathrm{s}$. If the clods is plastic, a running speed $v_{f}$ less than $4 \mathrm{~m} / \mathrm{s}$ can benefit the separation performance of potato-soil mixture ${ }^{[26]}$. Combined with the early harvest experiment, the running speed of the potato-soil mixture is about $v_{s}=1 \mathrm{~m} / \mathrm{s}$, and an optimal choice of running speed is $v_{f}=1.5-2.0 \mathrm{~m} / \mathrm{s}$.

\subsubsection{Vibration intensity adjusting device}

Potato harvester usually employs vibration technology to improve the separation performance. This vibration technology includes active and passive mode. Both vibration modes can improve the adaptability of harvesters, however, some studies also have shown it was one of the reasons causing potato damage and bruising $^{[9]}$. Vibration intensity is the key factor affecting the clod crushing and potato bruising performance. As the mixture of potato-soil transports, jitters and breaks on the sieve, some impurities gradually separate, as a consequence, the flow of potato-soil mixture is being in sparse form from dense. Therefore, the running speed of separation sieve, the structural parameters and the arrangement of the vibration device determine the movement characteristics of the separation sieve, which can affect the separation performance and harvest quality ${ }^{[27]}$. The vibration intensity of the separation sieve can be regulated by changing the degree of the top surface of the separation sieves (the tight segment) through the jitter rubber wheel during transport and separation. The vibration device is mainly composed of the jitter shaft, the fixing plate and jitter rubber wheel (Figure 3).

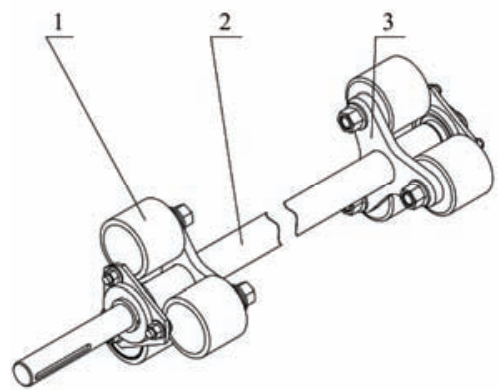

1. Jitter rubber wheel 2. Jitter shaft 3. Fixing plate

Figure 3 Structure diagram of vibration device

The vibration device is placed under middle area of the separation sieve's tight segment. A variable speed driving device II is installed on the end side of the jitter shaft that is passing through the side plate. As the separation sieve runs, the clods and impurities with diameter less than rod clearance $(34 \mathrm{~mm})$ gradually separates. According to the installation position of the front (rear) amplitude adjustment device and the requirement of the amplitude adjustment range, the diameter of single jitter rubber, the diameter of the circle that is defined by the centers of three jitter rubber wheels, the diameter of the effective working circle (externally tangent circle) of three jitter rubber wheels are $\phi_{d}=75 \mathrm{~mm}, \phi_{d y}=$ $133 \mathrm{~mm}$, and $\phi_{d g}=208 \mathrm{~mm}$, respectively. Since three rubber jitter wheels are placed against one fixing plate of the jitter shaft, one rotation of the vibration device is equivalent to three times reciprocating vibration on the surface of the separation sieve vertically, which facilitates the improvement of clod crushing performance on the sieve and separation of the potato-soil and impurities.

\subsection{Working principle and design parameters}

Before the experiment, the feeding device was filled up with the mixture of potato-soil according to the desired thickness and the length of the material layer. Once the driving device III/II are running steady, starting the driving device I and conveying the mixture of potato-soil to the separation sieve. The rotation speed of the driving device III can be changed through controlling the frequency converter. The vibration amplitude of different separation sieves can be obtained through adjusting the positions by the two-stage amplitude adjusting device. The vibration frequency of different separation sieves can be determined by adjusting the speed of the driving device II. The speed of different mixture of potato-soil on the feeding device can be adjusted by controlling the speed of driving device I. As the mixture of potato-soil are in transport, jittered and crushing on the sieve, the impurities such as soil and seedlings fall into impurity box, and the separated potatoes drop into receiving box at the same time with cushions passing through the guide slot. The main technical parameters are given in Table 1.

Table 1 Main technical parameters of test-bed for the separation of potato-soil

\begin{tabular}{lc}
\hline \multicolumn{1}{c}{ Technical parameters } & Value \\
\hline Overall dimensions $/ \mathrm{mm} \times \mathrm{mm} \times \mathrm{mm}$ & $1500 \times 800 \times 1026$ \\
Feeding device overall dimensions $/ \mathrm{mm} \times \mathrm{mm} \times \mathrm{mm}$ & $4500 \times 728 \times 952$ \\
Operating weight $/ \mathrm{kg}$ & 642 \\
Separation sieve $/ \mathrm{mm}$ & 800 \\
Distance of two adjacent rods $/ \mathrm{mm}$ & 45 \\
Separation sieve length $/ \mathrm{mm}$ & 3365 \\
Design bruising rate $/ \%$ & $\leq 2$ \\
\hline
\end{tabular}

On the basis of the preliminary results of the laboratory experiment and the field experiment of the potato harvester ${ }^{[25]}$, the parameters of the driving device of the test-bed are as follows, 1) Driving device I: the power is $1.5 \mathrm{~kW}$, speed ratio is 15 , rotational speed is $186 \mathrm{r} / \mathrm{min}$, roller diameter of conveyor belt is $155 \mathrm{~mm}$. 2) Driving device II: the power is $0.75 \mathrm{~kW}$, speed ratio is 12.5 , rotational speed is $225 \mathrm{r} / \mathrm{min}$. 3) Driving device III: power is $0.75 \mathrm{~kW}$, speed ratio is 12.5 , rotational speed is $225 \mathrm{r} / \mathrm{min}$.

\section{Working principle of impact recording and calibration of vibration intensity parameter}

\subsection{Impact recording system and working principle}

At the stage of separation, a proper collision and jump of the mixture can be conducive to separation performance, but when the impact velocity and the jumping height exceed the critical bruising 
and damage threshold, tangential bruising and other forms of damage can occur, leading to the reduction of the separation performance and harvest quality ${ }^{[28,29]}$. Therefore, the impact recording system (Figure 4), is employed to obtain the dynamic impact characteristics at different stages for detail data analysis.

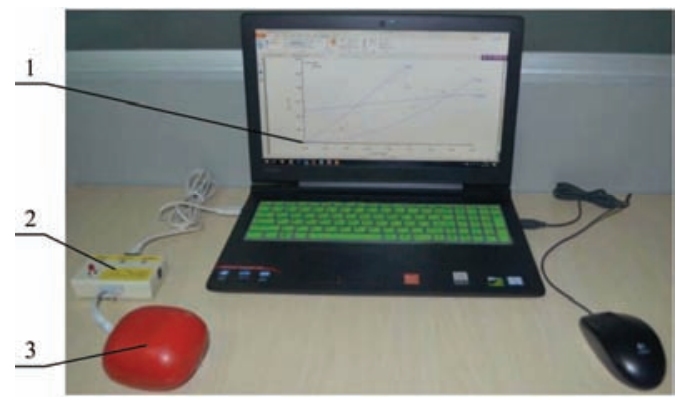

1. Computer 2. Ribbon cable 3. Test ball

Figure 4 Potato impact recording system

The impact recording system encompasses computer (software), ribbon cable and test ball. The test ball equips with three-dimensional acceleration sensor, power supply module, storage module, pressure sensor and single chip microcomputer, and a layer of hot-melt adhesive that is wrapped outside. The test ball is able to collect the impact characteristics through the three-dimensional acceleration sensor and pressure sensor effectively. The impact recording system could store and analyze the impact characteristics collected by the memory module and microprocessor. The test ball is buried in the potato-soil mixture during the experiment. It is assumed that the movement trajectory and the impact characteristics of the test ball can represent the actual behavior of potato-soil mixture. In this way, the impact characteristics can be detected during the whole process of potato-soil separation in real time. Meanwhile the PCIRD software is used for impact characteristics recording and data analysis. This software is allowed to setup parameters relevant to dynamic characteristics of the collision system in terms of the actual working condition. The acquired data can effectively reflect the start and end time of the collision. Once the data acquisition is finished, the PCIRD software can automatically generate various data reflecting dynamic characteristics of the collision system. Other than that, it could also provide the position information of the impact damage.

Before the experiment, the test ball was linked with computer by cable to set the recording data threshold $(20 \mathrm{~g})$. In the experiment, the test ball without ribbon cable was buried in the potato-soil mixture to record the impact data. Then, the driving device II, the driving device III and the driving device I were started sequentially. The potato-soil mixture was conveyed from the feeding device to the separation sieve. After the experiment, the test ball was linked with computer by ribbon cable to obtain the impact data and analyze by PCIRD software. In the process of potato-soil separation, the maximum impact acceleration, impact duration and velocity changes are the critical parameters affecting the harvest quality. The maximum impact acceleration can be calculated by the maximum acceleration of single impact, $G_{s p}=m_{s} a_{s}$, where $G_{s p}$ is maximum impact acceleration, $m_{s}$ is the mass of potato and $a_{s}$ is the acceleration. The velocity changes of single impact can be expressed as follows ${ }^{[30]}$ :

$$
\Delta v_{s p}=\int_{v_{p 2}}^{v_{p 1}} \mathrm{~d} v_{p}=\int_{t_{s p 1}}^{t_{s p 2}} a_{s} \mathrm{~d} t_{s p}
$$

where, $\Delta v_{s p}$ is the velocity changes of the impact process, $\mathrm{m} / \mathrm{s}$; $v_{p 1}$ is the velocity before the impact, $\mathrm{m} / \mathrm{s} ; v_{p 2}$ is the velocity after the impact, $\mathrm{m} / \mathrm{s} ; a_{s}$ is acceleration of impact process, $\mathrm{m} / \mathrm{s}^{2}$; and $t_{s p}$ is impact duration, $\mathrm{s}$.

Its impact energy can be determined as follows ${ }^{[23]}$.

$$
E_{s p}=m_{s}\left(\Delta v_{s p}\right)^{2} / 2
$$

where, $E_{s p}$ represents energy produced by impact, $\mathrm{J}$; while $m_{s}$ is mass of potatoes, $\mathrm{kg}$.

The bruising damage is related to the acceleration, contact area and duration of impact. The impact starts and ends when the calculated impact acceleration crosses $10 \%$ of the computed maximum $G_{s p}$ of the impact. The duration is the time interval determined by these two crossed points. The acceleration of the impact force gradually increases from zero to the maximum impact acceleration, and decreasing to zero gradually along with the time. The velocity change can be found by integrating the time interval defined by two crossed points when the computed impact acceleration of the impact crosses $10 \%$ of the computed maximum impact acceleration. The integral is numerically estimated by the trapezoidal method based on two adjacent points ${ }^{[20,31,32]}$. Velocity change is an important indicator characterizing the hardness of impact contact surface, and it is related to the energy absorption characteristics of impact surface.

The maximum impact acceleration not only comes from the rotation caused by separation sieve, but also comes from impacts among potatoes, rods, clods, stones and guide slot. When the vibration amplitude is large, the impact itself is also large which enables the test ball to record the impact signal with large amplitude due to collision where the maximum impact acceleration is sufficiently large. The signal of frequent impact and contact caused by the rolling process could also be collected when the vibration frequency of the separation sieve is high. In addition, when the running speed of the separation sieve changes, different impact characteristics are recorded at different initial velocity during the falling process as the test ball runs to the end of the separation sieve. It is shown that the trajectory of the potato-soil mixture is random to certain extent, and the direction and magnitude of the load are also quite different. In the separation process, it is difficult to determine the contact area during impact of potato-potato, potato-clod, potato-stone, and potato-rod ${ }^{[21]}$. The impact recording device uses the number of impacts, the maximum impact acceleration, velocity change and impact duration to investigate the effects of the separation parameters on the bruising damage, rather than through contact area.

\subsection{Calibration method and detection of vibration intensity parameters}

In order to accurately evaluate the effect of vibration intensity on the separation performance and harvest quality, five regions on the arc groove are determined by the positions of the front and rear amplitude adjustment handles. They are regions of $\mathrm{A}, \mathrm{B}, \mathrm{C}, \mathrm{D}$ and $\mathrm{E}$ (Figure 5). The vibration amplitude is related to the position of the front and rear amplitude adjustment handle in the arc groove, meanwhile the vibration frequency.

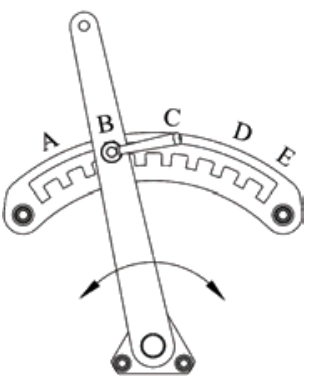

Figure 5 Region division of amplitude adjustment process 
Even if the front and rear amplitude adjustment handle is at the same position, the vibration amplitude of the separation sieve could still change as the vibration frequency alters. The separation sieve is too late to fall and hit separation sieve when the vibration frequency is too high. According to the design requirements of the separation device, the vibration frequency of the jitter rubber wheel ranges at 3-8 $\mathrm{Hz}^{[14]}$. The rotation speed of the jitter rubber wheel are $85 \mathrm{r} / \mathrm{min}, 95 \mathrm{r} / \mathrm{min}$ and $105 \mathrm{r} / \mathrm{min}$ in the calibration process respectively, and the corresponding vibration frequencies are $4.25 \mathrm{~Hz}, 4.75 \mathrm{~Hz}$ and $5.25 \mathrm{~Hz}$, respectively. For the purpose of accurately estimating the effects of the vibration amplitude on the experiment results for each vibration frequency, blank paper for recording the magnitude of the vibration amplitude is attached to the vertical plate on the side of the jitter rubber wheel. The resulting curve of the separation sieve can be generated in real time, reflecting the comprehensive effect on the separation sieves, the vibration frequency and the front and rear adjustment rubber wheel (Figure 6). The fluctuation range of the measured curve is the magnitude of vibration amplitude. The mean magnitude value can be found by three replications. The vibration frequency is calculated by the speed of the jitter shaft.

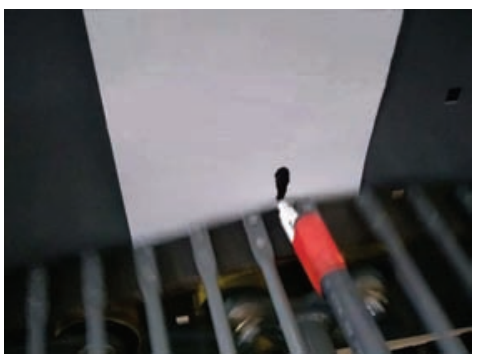

Figure 6 Calibration of vibration intensity

In order to investigate the effect of vibration frequency and amplitude on vibration intensity, three frequencies and five levels for the front and rear amplitude adjustment handle, resulting in seventy-five working conditions in total, are selected. In consideration of the comprehensive effect on separation performance and harvest quality due to vibration amplitude and frequency, the vibration effect produced by vibration adjusting device can be caused by following modes, low frequency and low amplitude vibration, high frequency and low amplitude vibration, medium frequency and medium amplitude vibration, low frequency and high amplitude vibration, high frequency and high amplitude. It includes five vibration intensity grades, which are I, II, III, IV and $\mathrm{V}$ in ascending order. The relevant parameters for different condition are given in Table 2. It is possible to find the maximum vibration intensity allowed based on the field experiment data through appropriate data analysis approach.

Table 2 Parameter adjustment and corresponding value under different vibration intensity conditions

\begin{tabular}{ccccc}
\hline $\begin{array}{c}\text { Vibration } \\
\text { intensity }\end{array}$ & Vibration form & Region-region & $\begin{array}{c}\text { Vibration } \\
\text { frequency/Hz }\end{array}$ & $\begin{array}{c}\text { Vibration } \\
\text { amplitude/mm }\end{array}$ \\
\hline I & $\begin{array}{c}\text { Low frequency low } \\
\text { amplitude }\end{array}$ & B-B & 4.25 & 20 \\
II & $\begin{array}{c}\text { High frequency low } \\
\text { amplitude }\end{array}$ & B-B & 5.25 & 20 \\
III & $\begin{array}{c}\text { Medium frequency } \\
\text { medium amplitude }\end{array}$ & A-D & 4.75 & 30 \\
IV & $\begin{array}{c}\text { Low frequency high } \\
\text { amplitude }\end{array}$ & E-B & 4.25 & 40 \\
V & $\begin{array}{c}\text { High frequency high } \\
\text { amplitude }\end{array}$ & E-B & 5.25 & 40 \\
\hline
\end{tabular}

The movement of the separation sieve in fact is simple harmonic motion forced by the jitter wheel. The amplitude is the maximum displacement of the separation sieve off its equilibrium position. Previous studies have already shown that the clods crushing performance on the separation sieve was directly proportional to the square of vibration amplitude and vibration frequency ${ }^{[14]}$. It can be found that the kinetic and potential energy of vibration intensity condition have a direct influence on the dynamic characteristics of the potato-soil mixture. In the other words, vibration intensity directly affects the separation performance, damage rate and bruising rate. It is found that the greater the vibration intensity is, the greater the mechanical energy produced by the separation sieve is, leading to a better separation performance of potato-soil mixture. On the other side, the deformation energy caused by the impacts, contacts and friction of the potatoes also increases, which is the main cause of the impact damage and tangent bruising ${ }^{[33,34]}$, as the mechanical energy of separation sieving increases. The early bench experiment has showed that the separation mode transitions through four stages, including smooth transport separation, low amplitude jitter, high amplitude jitter as the vibration intensity being stronger gently. Meanwhile the vibration frequency gradually increases (Figure 7). Through high-speed camera, the degrees of jumping and rolling, the transition into unstable state from stable movement were observed. Combined with the early field experiment of potato harvester, it is demonstrated that the calibration of vibration intensity in present work meets the requirement of field tests.

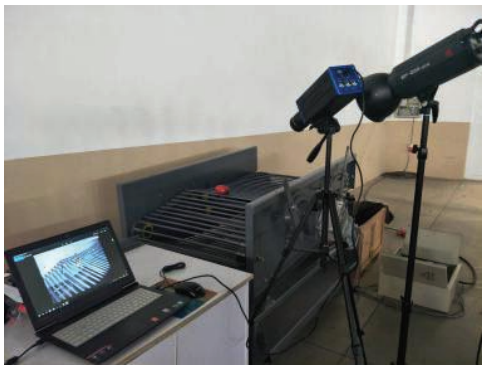

Figure 7 Bench experiment

\section{Materials and methods}

\subsection{Site and climate description}

The experimental plot locates in the Wang Machang Village, Jiehe town, Tengzhou City, Shandong Province. The mean annual temperature is $13.6^{\circ} \mathrm{C}$, the mean annual ground temperature is $16.3^{\circ} \mathrm{C}$, and the mean annual precipitation is $773.1 \mathrm{~mm}$. Deep ploughing depth was $30 \mathrm{~cm}$ before entering winter. Sprout planting was in middle and late February 2018 with ridge spacing of $70 \mathrm{~cm}$, ridge width of $50 \mathrm{~cm}$, ridge height of $25 \mathrm{~mm}$, planting spacing of $25 \mathrm{~cm}$, single row and double row planting. The potato planting pattern of "greenhouse + arch shed + plastic film" was adopted during the process of sowing. Once the seedling came out, the arch shed was removed. The greenhouse was removed in the rainy season (late April 2018). The field experiment was conducted from May 21th, 2018 to May 28th, 2018. The potato variety planted was the Xisen III. The seedlings were removed manually and the plastic film was removed before the experiment. The mean compaction at $0-15 \mathrm{~cm}$ soil depth was $58.33 \mathrm{kPa}$, the mean compaction at $15-30 \mathrm{~cm}$ soil depth was $432.56 \mathrm{kPa}$, and the mean moisture content at 0-30 cm soil depth was $13.05 \%$.

\subsection{Experiment instruments and equipment}

The experiment instruments and equipment include, 1) the potato impact recording device (the shell was covered with hot melt 
adhesive; the dimension is $80 \mathrm{~mm} \times 90 \mathrm{~mm} \times 60 \mathrm{~mm}$; the weight of test ball is $270 \mathrm{~g}$; sampling frequency is $488 \mathrm{~Hz}-5 \mathrm{kHz}$; memory is $32 \mathrm{~K}$; working environment temperature is $-29^{\circ} \mathrm{C}-60^{\circ} \mathrm{C}$, frequency response range from $5 \mathrm{~Hz}$ to $3 \mathrm{kHz}$; the maximum impact acceleration of the test ball is $450 \mathrm{~g},\left(\mathrm{~g}=9.8 \mathrm{~m} / \mathrm{s}^{2}\right)$; the device is produced by the Techmark company in the United States); 2) A high speed camera model with a Revealer 5F01 high-speed camera (image acquisition speed is $4 \mathrm{~GB} / \mathrm{s}$, The full picture is $2000 \mathrm{fps}$, produced by the Fuhuang Junda tech information technology company in Hefei city, Anhui Province; 3) Digital soil compaction meter with SPECTRUM SC-900 ranging from 0 to $7000 \mathrm{kPa}$; 4) The PCIRD software produced by the Techmark company in the United States; 4) Electric drying oven with DHG101-2 produced by the Hu Yue instrument and equipment company in Shangyu city, Zhejiang Province. Other than that, collection device of soil, the self-made separation test-bed of potato-soil, computer, tachometer and tape measure are also used in this work.

\subsection{Experiment method and experiment index}

In order to follow the digging depth under normal mechanized harvesting conditions, the conveyer belt of the feeding device is prepared such that it is covered with potato-soil mixture layer that has thickness of $4.5 \mathrm{~m}$ and width of $0.8 \mathrm{~mm}$. For data acquisition purpose, the test ball is buried in the mixture. According to predetermined parameters, the operator first starts driving device II /III. After separation sieve and jitter shaft both run stable, starting the driving device I. After each experiment is completed, the test ball is removed and the impact recording data is transferred to the computer. Experiment for each working condition has three replications for meaningful statistics. At the same time, the separation performance for each working condition is accounted for to comprehensively evaluate the separation performance of potato-soil ${ }^{[35]}$. The experiment records the movement characteristics of potato-soil mixture by high-speed camera, tracks the clods crushing process on the separation sieve. These data enable us to analyze the influence of the vibration intensity adjustment on clods crushing performance, and further make it possible to find the clods crushing mechanism under a variety of separation conditions.

With the planting pattern, potato varieties, thickness of mixture of potato-soil unchanged, the critical technical parameters affecting the separation performance and quality include feeding speed, running speed of separation sieve, vibration amplitude and vibration frequency. The preliminary results of early experiment have given a set of parameter range that could guarantee the harvest quality. These parameters are for the running speed of separation sieve, vibration amplitude and vibration frequency. The determined indexes used in this work include bruising rate and separation performance. For the sake of meeting the actual demand of the harvest condition, the angle of the separation sieve is $14^{\circ}$, and the running speed of conveyor belt is $1.0 \mathrm{~m} / \mathrm{s}$. For data analysis, the weight of the bruising potatoes, all harvested potatoes, and collected soil are measured. The bruising rate and separation performance are calculated through the following formula,

$$
\begin{aligned}
& \xi_{p}=\left[m_{p} /\left(m_{z s}\right)\right] \times 100 \% \\
& \xi_{f}=\left[m_{w f} /\left(m_{z h}\right)\right] \times 100 \%
\end{aligned}
$$

where: $\xi_{p}$ is bruising rate, $\% ; m_{p}$ is the weight of the bruising potatoes, $\mathrm{kg} ; m_{z s}$ is the total weight of the harvested potatoes, $\mathrm{kg}$; $\xi_{f}$ is separation performance of potato-soil, $\% ; m_{w f}$ is the weight of soil and impurity without separation, $\mathrm{kg} ; m_{z h}$ is the total weight of potato-soil mixture, $\mathrm{kg}$.

\section{Experiment design and result analysis}

In order to explore the influence of various parameters on the bruising rate and separation performance, meanwhile study the interaction among various factors, the field experiments (Figure 8) were conducted for two investigation purposes. 1) Investigating the impact characteristics during separation under different vibration intensities. High-speed cameras are employed to obtain the critical data describing the dynamics of potato-soil mixture. This enables us to observe in detail jumping and rolling of potatoes on clods crushing and separation. Combining with impact history data, the movement characteristics of potato-soil mixture can be studied systematically by varying parameters of interest. Thereby the influence of vibration intensity on harvest quality and separation performance can be found. 2) According to the experiment design principle of Box-Behnken, vibration amplitude, vibration frequency and running speed of the separation sieve are selected as the influence factors of the response surface analysis. The bruising rate and the separation performance are used as the evaluation indexes of the separation performance of the potato-soil mixture, while the maximum impact acceleration is treated as the model dependent variable.

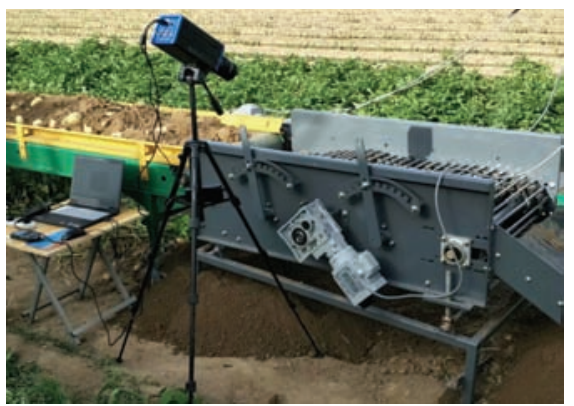

Figure 8 Field experiment of the separation of potato-soil

Based the preliminary experiment, meanwhile considering the characteristics of planting agronomy and soil, the running speed of separation sieve could be classified as low speed, medium speed and fast speed separation mode, all of which are within common speed range. The corresponding running speeds of the separation sieve driving shaft are $120 \mathrm{r} / \mathrm{min}, 140 \mathrm{r} / \mathrm{min}$ and $160 \mathrm{r} / \mathrm{min}$, respectively. The corresponding running speeds of the separation sieve are $1.54 \mathrm{~m} / \mathrm{s}, 1.80 \mathrm{~m} / \mathrm{s}$ and $2.06 \mathrm{~m} / \mathrm{s}$, respectively. Coding of experiment factors is given in Table 3.

Table 3 Coding of experiment factors

\begin{tabular}{cccc}
\hline \multirow{3}{*}{ Code } & \multicolumn{3}{c}{ Factor } \\
\cline { 2 - 4 } & $\begin{array}{c}\text { Vibration } \\
\text { amplitude } x_{1} / \mathrm{mm}\end{array}$ & $\begin{array}{c}\text { Vibration frequency } x_{2} \\
/ \mathrm{Hz}\end{array}$ & $\begin{array}{c}\text { Running speed of the } \\
\text { separation sieve } x_{3} / \mathrm{m}^{-1} \mathrm{~s}^{-1}\end{array}$ \\
\hline-1 & 20 & 4.25 & 1.54 \\
0 & 30 & 4.75 & 1.80 \\
1 & 40 & 5.25 & 2.06 \\
\hline
\end{tabular}

5.1 Potato-soil separation experiment under different vibration intensity conditions

In order to study the effect of potato-soil separation at five vibration intensity levels, the relevant experimental data are obtained through the experiment as provided in Table 4. The corresponding running speed of the separation sieve is $1.80 \mathrm{~m} / \mathrm{s}$.

As shown in Table 4, with the increasing of vibration amplitude levels from $\mathrm{I}$ to $\mathrm{V}$ with fixed running speed of the separation sieve, the number of impacts among potatoes correspondingly increases from one time to eleven times. Meanwhile, the maximum impact acceleration also increases to 
$115.79 \mathrm{~g}$ from $43.57 \mathrm{~g}$. As shown the average number of impacts and maximum impact acceleration of potato-soil mixture have increased significantly during the transition. In addition, the fluctuation range of velocity change and impact duration has increased noticeably. The bruising rate and the separation performance increases from $0.92 \%$ to $2.45 \%$ and from $87.3 \%$ to $99.7 \%$ respectively. Since the impact duration is in millisecond, it can be found that the impact among potatoes, between potato and bar, between potato and guide slot are collision process in time scale of millisecond. In fact, this is a complex dynamic system with mechanical energy exchange to certain extent. As indicated, the number of impacts and maximum impact acceleration of potato-soil mixture also promotes as the vibration intensity level increases. This benefits the crushing of the clods with a certain adhesive force, which is favorable for improving separation performance. However, the shear and friction caused by the impact contact make the bruising rate increased simultaneously. For the sake of maximizing the separation performance and harvest quality, it is therefore recommended that an optimal set of parameters should be selected according to the actual requirements.

Table 4 Movement characteristics and separation effect of potato-soil under different vibration intensities conditions

\begin{tabular}{|c|c|c|c|c|c|c|}
\hline \multirow{2}{*}{$\begin{array}{l}\text { Vibration intensity } \\
\text { levels }\end{array}$} & \multicolumn{4}{|c|}{ Movement characteristics } & \multicolumn{2}{|c|}{ Separation effect of potato- soil } \\
\hline & $\begin{array}{l}\text { Number of } \\
\text { impacts }\end{array}$ & $\begin{array}{l}\text { Maximum impact } \\
\text { acceleration } G_{s p} / g\end{array}$ & $\begin{array}{l}\text { Velocity changes } \Delta v_{s p} \\
\qquad / \mathrm{m} \cdot \mathrm{s}^{-1}\end{array}$ & $\begin{array}{l}\text { Impact duration } t_{s p} \\
\qquad / \mathrm{ms}\end{array}$ & $\underset{/ \%}{\operatorname{Bruising} \text { rate }} \xi_{p}$ & $\begin{array}{c}\text { Separation performance } \xi_{f} \\
/ \%\end{array}$ \\
\hline I & 1 & 43.57 & 1.58 & 4.1 & 0.52 & 87.3 \\
\hline II & 3 & 69.47 & $0.01-1.61$ & $0.26-6.14$ & 1.13 & 91.9 \\
\hline III & 6 & 74.24 & $0.49-1.71$ & $1.28-6.66$ & 1.31 & 94.2 \\
\hline $\mathrm{V}$ & 11 & 115.79 & $0.04-2.83$ & $1.02-9.47$ & 2.45 & 99.7 \\
\hline
\end{tabular}

The whole separation process of potato-soil mixture can be analyzed. 1) Low frequency and low amplitude separation. In this case, the separation sieve is running steady, therefore the mixture of potato-soil moves along with the separation sieve exactly. The impacts occur when the mixture of potato-soil falls to the cushion of guide slot, and its corresponding bruising rate is only $0.52 \%$. The main cause for bruising is the frictional and tangential force among potatoes and cushion. Since the mixture of potato-soil moves in a steady state, potatoes have no acceleration in perpendicular direction to the surface of separation sieve, consequently no clod crush due to vibration can occur. It is found that the separation performance is only $87.3 \%$ in experiment condition. In fact, low frequency and low amplitude separation is only suitable for sandy soil separation. 2) High frequency and low amplitude separation. On the contrary with low frequency and low amplitude separation, as the vibration frequency of jitter rubber wheel increases, and the vibration frequency increases up to three times, the mean maximum impact acceleration has increased by $59.4 \%$, the separation performance has increased from $87.3 \%$ to $91.9 \%$, but unfavorable bruising rate has increased by $117.3 \%$ as well. In this case a certain degree of vibration is produced in the potato-soil mixture. This vibration is beneficial to the crushing of clods similar to sandy soil where the cohesive force is small. 3) Medium frequency medium amplitude separation. When the potatoes move backward with the separation sieve, it could roll in the opposite direction of the separation sieve. This separation mode is suitable for potato-soil mixture in neutral soil. 4) Low frequency and high amplitude separation. As the vibration amplitude of the separation sieves increases, potato could roll backward and jump slightly. As a result, the clods crushing could also be improved. Therefore, it is suitable for the separation of potato-soil in the approximate sticky soil. 5) High frequency and high amplitude separation. In this case, the vibration amplitude and frequency both reach the maximum. As expected, it is beneficial to improve the separation performance and the clods crushing performance due to large vibration effect. Nevertheless, the maximum impact is $115.79 \mathrm{~g}$ for potato-soil mixture. It is found that the potatoes are prone to roll backward with the excitation of jitter rubber wheel. Meanwhile the potatoes with small mass could also have a big jump. Consequently, the rolling in opposite direction and the jumping in vertical direction could potentially add the time spending on the separation sieve for the mixture. To some extent, this is equivalent to extend the length of separation path. At the same time, the number of impacts measured by the test ball has reached to eleven times. In this mode, the separation performance is very high, $99.7 \%$, but the bruising rate is as high as $2.45 \%$. Even worse the harvest quality is not ideal either. This vibration intensity is probably only suitable for harvesting potato in the sticky soil. The analysis of 1)-5) hints that the smooth transport of potato-soil mixture gradually transitions into a state with rolling and jumping with the increase of vibration intensity level. Increasing the vibration amplitude could add significance of behavior rollover and reflux.

\subsection{Response surface analysis experiment}

According to the coding of experimental factors given in Table 3 , the experimental data of response surface can be constructed, hence performing relevant data analysis (Table 5). It can be seen that, except for experiment 8 , all the other experiments meet the requirements of the bruising rate less than $2 \%$ as stipulated in NY/T648-2015 Technical specification for quality evaluation of potato harvester. The velocity changes and impact duration can fluctuate within a certain range due to strong randomness within the system as the separation runs. In addition, due to its own viscoelastic characteristics, potatoes could experience both elastic and plastic deformation when the jitter rubber wheel exerts excitation and the separation sieve adds impact load ${ }^{[16,36]}$.

\subsubsection{Regression model establishment}

In the process of separation, the interaction with separation sieve and impact among potatoes and rods could contribute the frictional force, pressure and impact force. The impact force generated by the jitter rubber wheel is conducive to crushing and screening of the clods. In order to reflect the influence of various factors on the bruising rate and separation performance, the analysis of response surface mainly focuses on the effect of vibration amplitude, vibration frequency and running speed of the separation sieve on the maximum impact acceleration. The variance analysis of regression equation and the significance test was carried out. The Table 6 has given the results. The second order polynomial regression model of the maximum impact acceleration is expressed in the form of the code as follows, 


$$
G_{s p}=62.06+14.01 x_{1}+18.82 x_{2}+6.08 x_{3}-7.55 x_{1} x_{2}+3.43 x_{1} x_{3}+
$$$$
4.73 x_{2} x_{3}+16.72 x_{1}^{2}+7.08 x_{2}^{2}-2.99 x_{3}^{2}
$$

The $p$ value of the second order polynomial regression model obtained is less than 0.01 , meaning the maximum impact acceleration regression model is extremely significant (Table 6). The $p$ value of the lack of fit is more than 0.05 , the lack of fit is not significant, because of the $p$ value is more than 0.05 . Therefore, the second order polynomial regression equation could accurately reflect the relationship between the maximum impact acceleration and its dependent variables, including vibration amplitude, vibration frequency and running speed of the separation sieve.

Table 5 Analysis of response surface experiment

\begin{tabular}{|c|c|c|c|c|c|c|c|c|c|}
\hline \multirow{2}{*}{ Number } & \multirow{2}{*}{$\begin{array}{l}\text { Vibration } \\
\text { amplitude } x_{1} \\
\quad / \mathrm{mm}\end{array}$} & \multirow{2}{*}{$\begin{array}{l}\text { Vibration } \\
\text { frequency } x_{2} \\
\quad / \mathrm{Hz}\end{array}$} & \multirow{2}{*}{$\begin{array}{l}\text { Runing speed of the } \\
\text { separation sieve } x_{3} \\
\qquad / \mathrm{m} \cdot \mathrm{s}^{-1}\end{array}$} & \multicolumn{6}{|c|}{ Evaluating indicator } \\
\hline & & & & $\underset{\xi_{p} / \%}{\text { Bruising rate }}$ & $\begin{array}{c}\text { Separation } \\
\text { performance } \xi_{f} / \%\end{array}$ & $\begin{array}{l}\text { Number of } \\
\text { impacts }\end{array}$ & $\begin{array}{l}\text { Maximum impact } \\
\text { acceleration } G_{s p} / g\end{array}$ & $\begin{array}{l}\text { Velocity changes } \\
\Delta v_{s p} / \mathrm{m}^{-1} \mathrm{~s}^{-1}\end{array}$ & $\begin{array}{c}\text { Impact duration } \\
t_{s p} / \mathrm{ms}\end{array}$ \\
\hline 1 & 1 & 0 & -1 & 1.34 & 94.9 & 7 & 75.19 & $0.57-1.34$ & $3.07-5.89$ \\
\hline 2 & -1 & 0 & -1 & 1.20 & 91.4 & 6 & 56.52 & $0.43-1.92$ & $2.56-5.12$ \\
\hline 3 & 0 & -1 & -1 & 0.75 & 88.3 & 4 & 52.16 & $0.63-2.41$ & $5.12-7.17$ \\
\hline 4 & 1 & -1 & 0 & 1.46 & 95.1 & 7 & 87.52 & $0.63-1.52$ & $3.07-6.91$ \\
\hline 5 & 0 & 1 & -1 & 1.34 & 95.6 & 7 & 75.68 & $0.52-1.33$ & $2.56-7.42$ \\
\hline 6 & 0 & 0 & 0 & 1.31 & 92.6 & 6 & 60.67 & $0.01-2.96$ & $0.26-10.50$ \\
\hline 7 & 0 & 0 & 0 & 1.28 & 93.4 & 7 & 60.15 & $0.60-2.54$ & $0.26-8.45$ \\
\hline 8 & 1 & 1 & 0 & 2.33 & 99.3 & 11 & 114.71 & $0.40-2.16$ & $2.82-6.66$ \\
\hline 9 & 0 & 0 & 0 & 1.33 & 93.1 & 5 & 59.02 & $0.09-2.29$ & $1.79-6.91$ \\
\hline 10 & 0 & 1 & 1 & 1.74 & 97.5 & 6 & 89.59 & $0.05-2.02$ & $1.54-5.63$ \\
\hline 11 & -1 & 1 & 0 & 1.72 & 94.8 & 7 & 97.28 & $0.71-2.10$ & $3.84-9.47$ \\
\hline 12 & 0 & 0 & 0 & 1.36 & 93.5 & 5 & 64.76 & $0.63-1.13$ & $2.30-6.14$ \\
\hline 13 & -1 & -1 & 0 & 0.56 & 86.3 & 1 & 41.90 & $2.29-2.29$ & $7.94-7.94$ \\
\hline 14 & -1 & 0 & 1 & 1.34 & 93.1 & 6 & 69.52 & $1.25-2.35$ & $2.82-7.17$ \\
\hline 15 & 1 & 0 & 1 & 1.89 & 98.7 & 9 & 101.91 & $0.03-1.38$ & $0.26-6.40$ \\
\hline 16 & 0 & -1 & 1 & 0.97 & 87.5 & 5 & 47.15 & $0.53-1.84$ & $3.84-9.47$ \\
\hline 17 & 0 & 0 & 0 & 1.29 & 92.8 & 5 & 65.68 & $0.03-1.85$ & $0.51-6.66$ \\
\hline
\end{tabular}

Table 6 Variance analysis of regression equation

\begin{tabular}{cccccc}
\hline $\begin{array}{c}\text { Variation } \\
\text { sources }\end{array}$ & $\begin{array}{c}\text { Sum of } \\
\text { squares }\end{array}$ & Freedom & $\begin{array}{c}\text { Mean } \\
\text { square }\end{array}$ & $F$ & $P$ \\
\hline Model & 6416.75 & 9 & 712.97 & 24.18 & $0.0002^{* *}$ \\
$x_{1}$ & 1627.64 & 1 & 1627.64 & 55.20 & $0.0001^{* *}$ \\
$x_{2}$ & 2757.62 & 1 & 2757.62 & 93.52 & $<0.0001^{* *}$ \\
$x_{3}$ & 295.49 & 1 & 295.49 & 10.02 & $0.0158^{*}$ \\
$x_{1} x_{2}$ & 198.67 & 1 & 198.67 & 6.74 & $0.0356^{*}$ \\
$x_{1} x_{3}$ & 47.06 & 1 & 47.06 & 1.60 & 0.2469 \\
$x_{2} x_{3}$ & 89.49 & 1 & 89.49 & 3.03 & 0.1250 \\
$x_{1}{ }^{2}$ & 1141.91 & 1 & 1141.91 & 38.73 & $0.0004^{* *}$ \\
$x_{2}{ }^{2}$ & 196.32 & 1 & 196.32 & 6.66 & $0.0365^{*}$ \\
$x_{3}{ }^{2}$ & 31.59 & 1 & 31.59 & 1.07 & 0.3350 \\
Residual & 206.41 & 7 & 29.49 & & \\
Lack of fit & 171.19 & 3 & 57.06 & 6.48 & 0.0514 \\
Net error & 35.22 & 4 & 8.80 & & \\
Total deviation & 6623.16 & 16 & & & \\
\hline
\end{tabular}

Note: * * was extremely significant $(p<0.01)$, and * was significant $(0.01<p<$ $0.05)$.

As seen, the vibration amplitude and vibration frequency especially have an extremely significant impact on the maximum impact acceleration as both $p$ values are less than 0.01 . The running speed of the separation sieve has a more significant effect on the maximum impact acceleration than the others, while its $p$ value is less than 0.05 . In addition, the $x_{1} x_{2}$ of interaction term is significant, the two items $x_{1}^{2}$ has an extremely high effect in significance, and the two items $x_{2}^{2}$ has a more significant effect. Further analysis has shown that the order of each factor in terms of significance on the maximum impact acceleration is $x_{2}>x_{1}>x_{3}$, that is, vibration frequency $>$ vibration amplitude $>$ running speed of the separation sieve.

\subsubsection{Interaction analysis}

On the top of the analysis above, further analysis on the maximum impact acceleration acting on the test ball, and the response surface of vibration frequency, vibration amplitude and running speed of the separation sieve on the maximum impact acceleration are performed and the results are generated through Design-Expert 8.0.6 software (Figures 9-11). The maximum impact acceleration could increase significantly with the vibration frequency increases from $4.25 \mathrm{~Hz}$ to $5.25 \mathrm{~Hz}$ with the vibration amplitude unchanged. With the vibration frequency rises, the number of impacts among potatoes is grown and the movement state of potato gradually transforms to unstable, simultaneously.

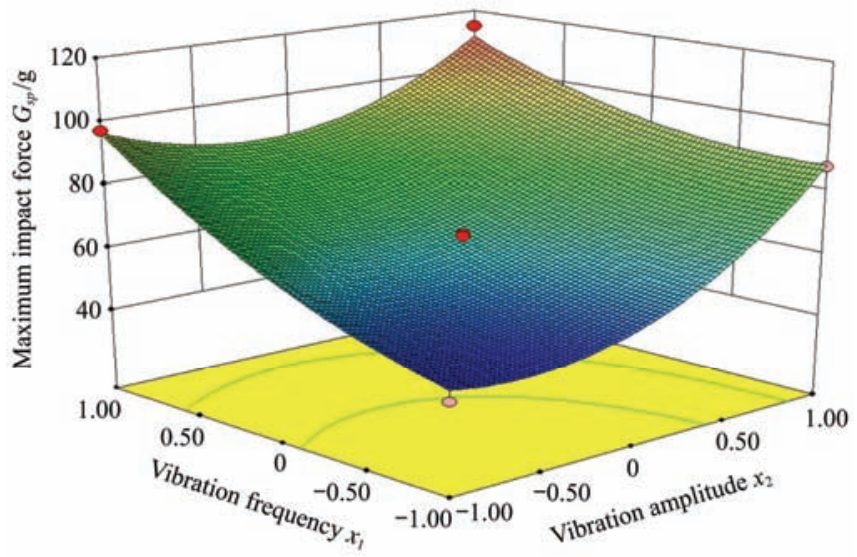

Figure 9 Effect of vibration amplitude and vibration frequency on maximum impact acceleration 


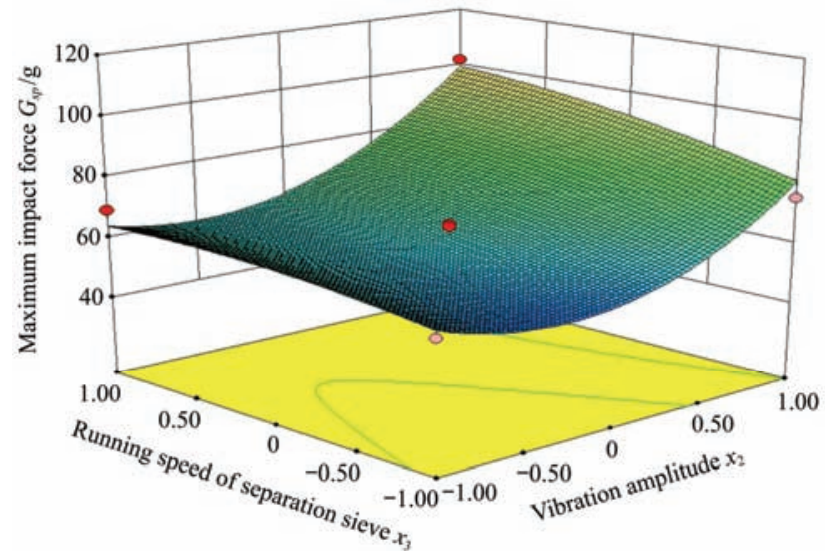

Figure 10 Effect of vibration amplitude and running speed of the separation sieve on maximum impact acceleration

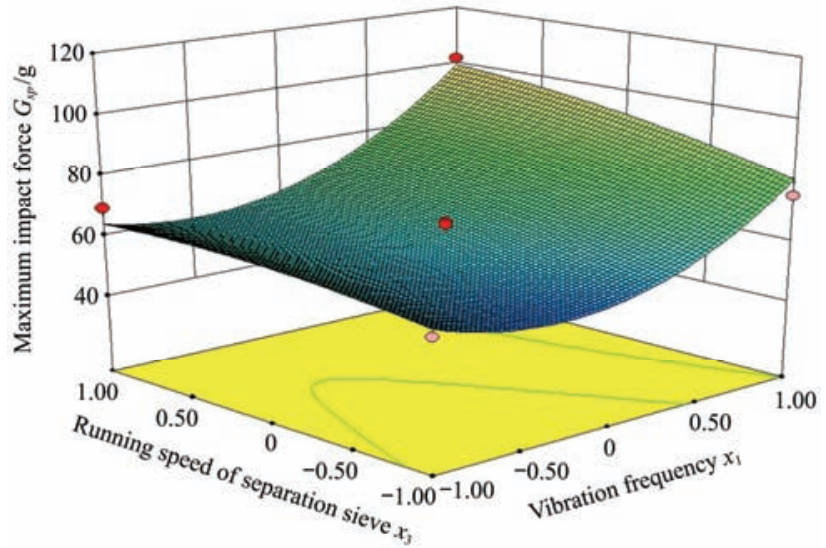

Figure 11 Effect of vibration frequency and running speed of the separation sieve on maximum impact acceleration

Consequently, the separation performance increases as the vibration frequency increases, however, the unfavorable bruising rate of potatoes also grow. This observation is consistent with the results of $\mathrm{Li}$ Tao et al. ${ }^{[33]}$ The maximum impact acceleration increases significantly with the vibration amplitude increases from $20 \mathrm{~mm}$ to $40 \mathrm{~mm}$ while the vibration frequency is fixed. With the increasing of vibration amplitude, the jumping height of potatoes also increases significantly. And it is found that more and more potatoes attempt to roll on the surface of separation sieve. Similarly, the separation performance increases with the increase of vibration amplitude, however, the bruising rate also increases. It has been seen that the maximum impact acceleration increases slowly with the increase in the running speed of the separation sieve from $1.54 \mathrm{~m} / \mathrm{s}$ to $2.06 \mathrm{~m} / \mathrm{s}$ with the vibration frequency or vibration amplitude unchanged. With the increase of running speed of the separation sieve, the vibration due to the movement of the plum blossom wheel turns strong and the dynamic load caused by the motion heterogeneity is being large. In addition, the vibration intensity of the jitter rubber wheel also has an indirect effect on the movement of the potato-soil mixture. The vibration of jitter rubber wheel could cause vibration of separation sieve, thereafter potentially change dynamics of potato-soil mixture. The influence of vibration frequency or vibration amplitude of the jitter rubber wheel on the maximum impact acceleration is similar as that of separation sieve when its running speeding is unchanged.

As observed, it can be concluded that the effect of the running speed of the separation sieve on the maximum impact acceleration is less significant than the vibration amplitude and vibration frequency forced by jitter rubber wheel. Therefore, the separation performance and bruising rate can be mainly determined by the vibration amplitude and vibration frequency.

\subsubsection{Parameter optimization and verification}

To ensure that all experiments could meet the requirements of the bruising rate less than $2 \%$ as stipulated in NY/T648-2015 Technical specification for quality evaluation of potato harvester and reach a good separation performance, as indicated in Table 5, the maximum collision force that can meet the above requirements is $101.91 \mathrm{~g}$. For minimizing the bruising rate, the optimal operation parameters obtained through the regression model are as follows, the vibration amplitude is $34.1 \mathrm{~mm}$, the vibration frequency is $5.24 \mathrm{~Hz}$, and the running speed of the separation sieve is $2.05 \mathrm{~m} / \mathrm{s}$. The mean value of the parameters obtained from three validation experiments are as follows, the maximum collision force is $98.62 \mathrm{~g}$, the relative error is $3.23 \%$, the bruising rate is $1.81 \%$, and the separation performance is $98.5 \%$. This could completely meet the requirements of NY/T648-2015 Technical specification for quality evaluation of potato harvester. It is demonstrated that the regression model is reliable in this application and it has the capability to predict experiment results and reflect the mechanism of the maximum impact acceleration in the actual condition of harvesting.

\section{Conclusions}

1) The mean number of impacts and maximum impact acceleration were affected significantly by the vibration intensity. It was found that as the level of vibration intensities gradually increased from $\mathrm{I}$ to $\mathrm{V}$, as a response the number of impacts also grew from 1 to 11, and the maximum impact acceleration increased from $43.57 \mathrm{~g}$ to $115.79 \mathrm{~g}$. Similarly, the movement of mixture of potato-soil gradually transitions into unsteady state as the vibration intensity being stronger. In addition, the bruising rate increased from $0.92 \%$ to $2.45 \%$, and the separation performance increased from $87.3 \%$ to $99.7 \%$ along with the vibration intensity rising.

2) A mathematical model establishing the relationship between maximum impact acceleration and dependent variables, including vibration amplitude, vibration frequency and running speed of the separation sieve is constructed. The significance of each factor on the maximum impact acceleration in descending order was vibration frequency, vibration amplitude, running speed of the separation sieve, that is $x_{2}>x_{1}>x_{3}$. The best performance could be obtained under the optimum operating parameters with vibration amplitude of $34.1 \mathrm{~mm}$, vibration frequency of $5.24 \mathrm{~Hz}$, and running speed of the separation sieve of $2.05 \mathrm{~m} / \mathrm{s}$. Under this combination of parameters, the maximum impact acceleration was $98.62 \mathrm{~g}$, relative error was $3.23 \%$, bruising rate was $1.81 \%$ and the separation performance was $98.5 \%$.

\section{Acknowledgements}

This research was supported by the Program for National Key Research and Development Plan (2016YFD0701603-02), Shandong Taishan Industry Leading Talent Project (LJNY201615), and Shandong Province Major Science and Technology Innovation Project (2017CXGC0219), and Innovative Research Team in University of China (IRT13039), Shandong Province Agricultural Machinery Equipment Research and Development and Innovation Project (2016YF034). 


\section{[References]}

[1] Koga N, Kajiyama T, Senda K, Iketani S, Tamiya S, Tsuda, S. Energy efficiency of potato production practices for bioethanol feedstock in northern Japan. European Journal of Agronomy, 2013; 44(1): 1-8.

[2] Kang W Q, Fan M S, Ma Z, Shi X H, Zheng H L. Luxury absorption of potassium by potato plants. American Journal of Potato Research, 2014; 91(5): 573-578.

[3] Xu J F, Jin L P. Advances and perspectives in research of potato genetics and breeding. Scientia Agricultura Sinica, 2017; 50(6): 990-1015.

[4] Zhou K, Jensen A L, Bochtis D D, Sorensen C G. Simulation model for the sequential in-field machinery operations in a potato production system. Computers \& Electronics in Agriculture, 2015; 116(8): 173-186.

[5] Dai F, Guo X H, Zhao W Y, Xin S L, Liu X L, Wu Z W. Design and experiment of canvas belt combined operation machine for potato digging and plastic film collecting. Transactions of the CSAM, 2018; 49(3): 104-113. (in Chinese)

[6] Wu J M, Li H, Sun W, Huang X P, Zhang W. Experiment on poke finger wheel type potato digger. Transactions of the CSAE, 2011; 27(7): 173-177. (in Chinese)

[7] Devsh K, Ashok T. Performance evaluation of tractor drawn potato digger cum-elevator. International Journal of Agricultural Science and Research, 2017; 7(2): 433-448.

[8] Nappe Mordi N. Al-Dosary. Potato harvester performance on tubers damage at the eastern of Saudi Arabia. CIGR Journal, 2016; 18(2): $32-42$.

[9] Lü J Q, Tian Z E, Yang Y, Shang Q Q, Wu J E. Design and experimental analysis of 4U2A type double-row potato digger. Transactions of the CSAE, 2015; 31(6): 17-24. (in Chinese)

[10] Wei H A, Wang D, Lian W X, Shao S L, Yang X P, Huang X P. Development of 4UFD-1400 type potato combine harvester. Transactions of the CSAE, 2013; 29(1): 11-17. (in Chinese)

[11] Yang R B, Yang H G, Shang S Q, Xu P X, Cui G P, Liu L H. Design and test of poking roller shoving type potato harvester. Transactions of the CSAM, 2016; 47(7): 119-126.

[12] Azizi P, Dehkordi N S, Farhadi R. Design, construction and evaluation of potato digger with rotary blade.. Cercetari Agronomice in Moldova, 2014; 47(3): 5-13.

[13] Lü J Q, Shang Q Q, Yang Y, Li Z H, Li J C, Liu Z Y. Design optimization and experiment on potato haulm cutter. Transactions of the CSAM, 2016; 47(5): 106-114. (in Chinese)

[14] Lü J Q, Sun H, Dui H, Peng M M, Yu J Y. Design and experiment on conveyor separation device of potato digger under heavy soil condition. Transactions of the CSAM, 2017; 48(11): 146-155. (in Chinese)

[15] Yang R B, Yang H G, Shang S Q, Ni Z W, Liu Z S, Guo D. Design and experiment of vertical circular separating and conveying device for potato combine harvester. Transactions of the CSAE, 2018; 34(3): 10-18. (in Chinese)

[16] Feng B, Sun W, Shi L R, Sun B G, Zhang T, Wu J M. Determination of restitution coefficient of potato tubers collision in harvest and analysis of its influence factors. Transactions of the CSAE, 2017; 33(13): 50-57.

[17] Opara U L, Pathare P B. Bruise damage measurement and analysis of fresh horticultural produce: A review. Postharvest Biology \& Technology, 2014; 91(5): 9-24

[18] Tvan C, Tijskens E, Ramon H, Verschoore R, Sonck B. Characterisation of a potato-shaped instrumented device. Biosystems Engineering, 2003;
86(3): $275-285$.

[19] Dai F, Zhao W Y, Sun W, Wu Z W, Song X F, Li Y. Design and Experiment of combined operation machine for potato harvesting and plastic film pneumatic auxiliary collecting. Transactions of the CSAM, 2017; 48(1): 64-72. (in Chinese)

[20] Yu P, Li C, Rains G, Hamrita T. Development of the berry impact recording device sensing system: software. Computers \& Electronics in Agriculture, 2011; 77(2): 195-203.

[21] Du X Q, Li D W, He L Y, Wu C Y, Lin L P. Fruit motion analysis in process of mechanical vibration harvesting based on electronic fruit technique. Transactions of the CSAE, 2017; 33(17): 58-64.

[22] Zeebroeck M V, Linden V V, Ramon H, De Baerdemaeker J, Nicolaï E B, Tijskens M. Impact damage of apples during transport and handling. Postharvest Biology \& Technology, 2007; 45(2): 157-167.

[23] Canneyt T V, Tijskens E, Ramon H, Verschoore R, Sonck B Development of a predictive tissue discolouration model based on electronic potato impacts. Biosystems Engineering, 2004; 88(1): 81-93.

[24] Robertp S, Edwardc L. Immunocytological comparison of native and wound periderm maturation in potato tuber. American Journal of Potato Research, 2004; 81(2): 119-124.

[25] Wei Z C, Li H W, Sun C Z, Li X Q, Liu W Z, Su G L, et al. Improvement of potato harvester with two segment of vibration and wave separation. Transactions of the CSAE, 2018; 34(12): 42-52.

[26] Chinese Academy of Agricultural Mechanization Sciences. Manual of agricultural machinery design. Beijing: China Agricultural Technology Press, 2007. (in Chinese)

[27] Shi L R, Wu J M, Zhao W Y, Sun W, Wang D, Li H, et al. Design and experiment on potato digger of disc ce-grate type. Transactions of the CSAE, 2012; 28(24): 15-21. (in Chinese)

[28] Praeger U, Surdilovic J, Truppel I, Herold, B, Geyer, M. Comparison of electronic fruits for impact detection on a laboratory scale. Sensors, 2013; 13(6): 7140-7155.

[29] Xie S S, Wang C G, Deng W G, Li X, Qi S H. Separating mechanism analysis and parameter optimization experiment of swing separation sieve for potato and soil mixture. Transactions of the CSAM, 2017; 48(11): 156-164. (in Chinese)

[30] Van Canneyt T, Langenakens, J, Tijskens E, Ramod H. Characterization of a potato-shaped instrumented tool to predict mechanical damage to potatoes. Ifac Proceedings Volumes, 2001; 34(28): 61-66.

[31] Yu P C, Li C Y, Glen Rains, Hamrita, T. Development of the berry impact recording device sensing system: hardware design and calibration. Computers and Electronics in Agriculture, 2011; 79(2): 103-111.

[32] www.techmark-inc.com.

[33] Li T, Zhou J, Xu W Y, Li Q L, Zhang H, Qin X T, et al. Development of 4UGS2 type double-row sweet potato harvester. Transactions of the CSAE, 2018; 34(11): 26-33.

[34] Bao Y D, Yang C, Zhao Y L, Liu X L, Guo Y L. Collision injury assessment of mechanical harvesting blueberry fruit based on collision deformation energy. Transactions of the CSAE, 2017; 33(16): 283-292.

[35] NY/T648 - 2015 Technical specification of quality evaluation for potato harvesters. Beijing: China Standard Press, 2015. (in Chinese)

[36] Lopez-Maestresalas A, Keresztes J C, Goodarzi M, Arazuri S, Jaren C, Saeys W. Non-destructive detection of blackspot in potatoes by Vis-NIR and SWIR hyperspectral imaging. Food Control, 2016; 70: 229-241. 